\title{
Efficient Techniques for Solving the Periodic Projected Lyapunov Equations and Model Reduction of Periodic Systems
}

\author{
Mohammad-Sahadet Hossain and M. Monir Uddin \\ Department of Mathematics and Physics, North South University, Dhaka, Bangladesh \\ Correspondence should be addressed to M. Monir Uddin; monir.uddin@northsouth.edu
}

Received 8 September 2016; Revised 17 January 2017; Accepted 22 January 2017; Published 14 February 2017

Academic Editor: Masoud Hajarian

Copyright ( 2017 Mohammad-Sahadet Hossain and M. Monir Uddin. This is an open access article distributed under the Creative Commons Attribution License, which permits unrestricted use, distribution, and reproduction in any medium, provided the original work is properly cited.

\begin{abstract}
We have presented the efficient techniques for the solutions of large-scale sparse projected periodic discrete-time Lyapunov equations in lifted form. These types of problems arise in model reduction and state feedback problems of periodic descriptor systems. Two most popular techniques to solve such Lyapunov equations iteratively are the low-rank alternating direction implicit (LR-ADI) method and the low-rank Smith method. The main contribution of this paper is to update the LR-ADI method by exploiting the ideas of the adaptive shift parameters computation and the efficient handling of complex shift parameters. These approaches efficiently reduce the computational cost with respect to time and memory. We also apply these iterative Lyapunov solvers in balanced truncation model reduction of periodic discrete-time descriptor systems. We illustrate numerical results to show the performance and accuracy of the proposed methods.
\end{abstract}

\section{Introduction}

Periodic systems and control theory have received a lot of attention in many areas of science and engineering, especially in the areas where the periodic control is deserved, such as spacecraft attitude control, control of industrial processes and mechanical systems, helicopter design, and nonlinear circuit design [1-6]. In this paper, we consider the linear periodic discrete-time descriptor systems with time-varying dimensions in the form

$$
\begin{aligned}
E_{k} x_{k+1} & =A_{k} x_{k}+B_{k} u_{k}, \\
y_{k} & =C_{k} x_{k},
\end{aligned}
$$

$$
k \in \mathbb{Z}
$$

where $E_{k} \in \mathbb{R}^{\mu_{k+1} \times n_{k+1}}, A_{k} \in \mathbb{R}^{\mu_{k+1} \times n_{k}}, B_{k} \in \mathbb{R}^{\mu_{k+1} \times m_{k}}, C_{k} \in$ $\mathbb{R}^{q_{k} \times n_{k}}$ are periodic with a period $K \geq 1, \sum_{k=0}^{K-1} \mu_{k}=\sum_{k=0}^{K-1} n_{k}=$ $n, \sum_{k=0}^{K-1} m_{k}=m$, and $\sum_{k=0}^{K-1} q_{k}=q$. If all the $E_{k}$ matrices are nonsingular, then (1) can be transformed into a periodic standard system.

Model reduction means to replace the given mathematical model (1) by a much smaller model which preserves certain crucial properties of the original model, such as stability and passivity, and it is then amenable for simulation and analysis. Analysis and reduced-order modeling of system (1) may require solving large-scale sparse projected periodic discrete-time Lyapunov equations. Model reduction procedures of such periodic systems using direct solution approaches have been considered in [6-10]. Recently, attention has been devoted to iterative solutions of large-scale sparse Lyapunov equations using the alternating directions implicit (ADI) method [11, 12], the Smith method [12-14], and Krylov subspace methods $[15,16]$. Generalization of all these methods has also been considered in $[17,18]$.

However, low-rank iterative methods and their application in model reduction of such periodic descriptor systems have been considered in $[19,20]$. The drawbacks of the ADI method proposed that there are the suboptimal shifts computations and the slow convergence of the iterative solutions to the exact solutions. In this paper, we proposed the adaptive shift parameters [21] computation instead of suboptimal parameters and explored the efficient handing of the complex shift [22] parameters. These two proposed schemes reduce the computational cost and fasten the convergence of the 
iterative solutions to exact solutions. Numerical results are discussed to show the efficiency of the techniques.

The paper outline is as follows. In Section 2, we briefly review the analogous time-invariant representation of the periodic systems, known as lifted representation of periodic systems. We discuss the cyclic lifted representation of periodic descriptor system (1). We reviewed the causal-noncausal decomposition, the periodic projected Lyapunov equations [8], and their corresponding lifted forms [7]. We also review some important concepts of the discrete-time descriptor system in linear time-invariant (LTI) form and the balanced truncation (BT) model reduction in LTI case. We also discuss the basics of the generalized ADI method in this section. The main idea behind this is that the concepts in the LTI structures will help more precisely to understand their corresponding periodic interpretations. In Section 3, we discuss the efficient solution of the causal lifted Lyapunov equations using the adaptive shifts based LR-ADI method. We also reviewed the Smith method from [20] for noncausal lifted Lyapunov equations in Section 4. We consider a balanced truncation model reduction method for periodic descriptor systems in Section 5. Section 6 contains numerical results that illustrate the efficiencies of the described iterative methods in model reduction of periodic descriptor system. Section 7 deficits the conclusion and remarks.

\section{Preliminaries}

In this section, we review the analogous time-invariant representation of the periodic systems, known as lifted representation. We also introduce some notations and briefly review the fundamental concept of balanced truncation model reduction for LTI discrete-time systems. We, afterward, generalize these concepts and results in the periodic setting.

2.1. Cyclic Lifted Representation of Periodic Systems. Analysis and modeling of periodic discrete-systems are often described by an analogous time-invariant representation of the periodic systems, known as lifted representation $[1,2,6]$. Here we consider the cyclic lifted representation [4] of periodic descriptor system (1), given by

$$
\begin{aligned}
\mathbf{E X}_{k+1} & =\mathbf{A X}_{k}+\mathbf{B U}_{k}, \\
\mathbf{Y}_{k} & =\mathbf{C X}_{k},
\end{aligned}
$$

where

$$
\begin{aligned}
& \mathbf{E}=\operatorname{diag}\left(E_{0}, E_{1}, \ldots, E_{K-1}\right), \\
& \mathbf{B}=\operatorname{diag}\left(B_{0}, B_{1}, \ldots, B_{K-1}\right), \\
& \mathbf{A}=\left[\begin{array}{cccc}
0 & \ldots & 0 & A_{0} \\
A_{1} & & 0 \\
& \ddots & & \vdots \\
0 & & A_{K-1} & 0
\end{array}\right],
\end{aligned}
$$

$$
\mathbf{C}=\left[\begin{array}{ccccc}
0 & & \cdots & 0 & C_{0} \\
C_{1} & & & & 0 \\
& \ddots & & & \vdots \\
0 & & & C_{K-1} & 0
\end{array}\right] .
$$

The descriptor vector, system input, and output of (2) are related to those of (1) via

$$
\begin{aligned}
& \mathbf{X}_{k}=\left[\begin{array}{c}
x_{1} \\
\vdots \\
x_{K-1} \\
x_{0}
\end{array}\right] \text {, } \\
& \mathbf{U}_{k}=\left[\begin{array}{c}
u_{0} \\
u_{1} \\
\vdots \\
u_{K-1}
\end{array}\right] \text {, } \\
& \mathbf{Y}_{k}=\left[\begin{array}{c}
y_{0} \\
y_{1} \\
\vdots \\
y_{K-1}
\end{array}\right] \text {, }
\end{aligned}
$$

respectively. We can define the important concepts and dynamics of the periodic systems, such as regularity and asymptotic stability, by using their corresponding lifted forms.

2.2. The PPDLEs and Their Lifted Forms. Consider periodic discrete-time descriptor system (1), where the set of periodic matrix pairs $\left\{\left(E_{k}, A_{k}\right)\right\}_{k=0}^{K-1}$ is periodic stable [20, 23]. Then the periodic Gramians of discrete-time periodic descriptor systems satisfy a class of projected discrete-time Lyapunov equations $[7,8]$.

Theorem 1 (see [23]). (1) The periodic projected discrete-time Lyapunov equations with special right hand sides

$$
\begin{gathered}
A_{k} G_{k}^{c r} A_{k}^{T}-E_{k} G_{k+1}^{c r} E_{k}^{T}=-P_{l}(k) B_{k} B_{k}^{T} P_{l}(k)^{T}, \\
G_{k}^{c r}=P_{r}(k) G_{k}^{c r} P_{r}(k)^{T}, \\
A_{k} G_{k}^{n c r} A_{k}^{T}-E_{k} G_{k+1}^{n c r} E_{k}^{T}=Q_{l}(k) B_{k} B_{k}^{T} Q_{l}(k)^{T}, \\
G_{k}^{n c r}=Q_{r}(k) G_{k}^{n c r} Q_{r}(k)^{T},
\end{gathered}
$$

with $k=0,1, \ldots, K-1$, have the unique symmetric, positive semidefinite periodic solutions $G_{k}^{c r}$ and $G_{k}^{n c r}$, respectively. 
(2) The periodic projected discrete-time Lyapunov equations with special right hand sides

$$
\begin{aligned}
A_{k}^{T} G_{k+1}^{c o} A_{k}-E_{k-1}^{T} G_{k}^{c o} E_{k-1} & =-P_{r}(k)^{T} C_{k}^{T} C_{k} P_{r}(k), \\
G_{k}^{c o} & =P_{l}(k-1)^{T} G_{k}^{c o} P_{l}(k-1), \\
A_{k}^{T} G_{k+1}^{n c o} A_{k}-E_{k-1}^{T} G_{k}^{n c o} E_{k-1} & =Q_{r}(k)^{T} C_{k}^{T} C_{k} Q_{r}(k), \\
G_{k}^{n c o}= & Q_{l}(k-1)^{T} G_{k}^{n c o} Q_{l}(k-1),
\end{aligned}
$$

with $k=0,1, \ldots, K-1$, have the unique symmetric, positive semidefinite periodic solutions $G_{k}^{c o}$ and $G_{k}^{n c o}$, respectively.

The solutions $\left\{G_{k}^{\mathrm{cr}}\right\}_{k=0}^{K-1}$ of (5) and $\left\{G_{k}^{\mathrm{ncr}}\right\}_{k=0}^{K-1}$ of (6), respectively, are called the causal and noncausal reachability Gramians of system (1). Similarly, the solutions $\left\{G_{k}^{\mathrm{co}}\right\}_{k=0}^{K-1}$ and $\left\{G_{k}^{\text {nco }}\right\}_{k=0}^{K-1}$ of (7) and (8), respectively, are called the causal and noncausal observability Gramians of (1).

Theorem 2 (see [7]). Consider a periodic descriptor system (1) and the corresponding lifted form (2), where $\left\{\left(E_{k}, A_{k}\right)\right\}_{k=0}^{K-1}$ is periodic stable. Then the matrices $\mathbf{G}^{c r}=\operatorname{diag}\left(G_{1}^{c r}, \ldots\right.$, $\left.G_{K-1}^{c r}, G_{0}^{c r}\right)$ and $\mathbf{G}^{n c r}=\operatorname{diag}\left(G_{1}^{n c r}, \ldots, G_{K-1}^{n c r}\right)$ solve the lifted projected discrete-time algebraic Lyapunov equations (LPDALEs):

$$
\begin{array}{r}
\mathbf{A G}^{c r} \mathbf{A}^{T}-\mathbf{E G}^{c r} \mathbf{E}^{T}=-\mathbf{P}_{l} \mathbf{B B}^{T} \mathbf{P}_{l}^{T}, \quad \mathbf{G}^{c r}=\mathbf{P}_{r} \mathbf{G}^{c r} \mathbf{P}_{r}^{T}, \\
\mathbf{A G}^{n c r} \mathbf{A}^{T}-\mathbf{E G}^{n c r} \mathbf{E}^{T}=\mathbf{Q}_{l} \mathbf{B} \mathbf{B}^{T} \mathbf{Q}_{l}^{T}, \\
\mathbf{G}^{n c r}=\mathbf{Q}_{r} \mathbf{G}^{n c r} \mathbf{Q}_{r}^{T},
\end{array}
$$

respectively, where $\mathbf{E}, \mathbf{A}$, and $\mathbf{B}$ are as in (3), and

$$
\begin{aligned}
& \mathbf{P}_{l}=\operatorname{diag}\left(P_{l}(0), P_{l}(1), \ldots, P_{l}(K-1)\right), \\
& \mathbf{Q}_{l}=I-\mathbf{P}_{l}, \\
& \mathbf{P}_{r}=\operatorname{diag}\left(P_{r}(1), \ldots, P_{r}(K-1), P_{r}(0)\right), \\
& \mathbf{Q}_{r}=I-\mathbf{P}_{r} .
\end{aligned}
$$

The matrices $\mathbf{G}^{\mathrm{cr}}$ and $\mathbf{G}^{\mathrm{ncr}}$ are the solutions of the lifted causal and noncausal reachability Gramians, respectively. A similar result can also be stated for the lifted causal and noncausal observability Gramians [7]. The solution techniques for such Lyapunov equations are shown in [20] using lowrank ADI methods. In the following section we mainly focus on the solution techniques of (9a) more efficiently.

2.3. Balanced Truncation for LTI Discrete-Time Systems. In order to understand the BT model reduction method for the time-varying discrete-time systems, we briefly review the idea for the time-invariant case. Consider a LTI discrete-time system:

$$
\begin{aligned}
\bar{E} \bar{x}_{k+1} & =\bar{A} \bar{x}_{k}(t)+\bar{B} u_{k}, \\
\bar{y}_{k} & =\bar{C} \bar{x}_{k},
\end{aligned}
$$

in which $\bar{E} \in \mathbb{R}^{n_{c} \times n_{c}}$ is nonsingular, and $\bar{A} \in \mathbb{R}^{n_{c} \times n_{c}}$, $\bar{B} \in \mathbb{R}^{n_{c} \times m_{c}}$, and $\bar{C} \in \mathbb{R}^{q_{c} \times n_{c}}$. Assume that the original system is asymptotically stable; that is, all finite eigenvalues of the system pencil lie inside the unite circle; then the controllability Gramian $P \in \mathbb{R}^{n_{c} \times n_{c}}$ and the observability Gramian $Q \in \mathbb{R}^{n_{c} \times n_{c}}$ can be computed by solving the discretetime Lyapunov equations (see, e.g., [24]):

$$
\begin{aligned}
& \bar{A} P \bar{A}^{T}-\bar{E} P \bar{E}^{T}=-\bar{B} \bar{B}^{T}, \\
& \bar{A}^{T} Q \bar{A}-\bar{E}^{T} Q \bar{E}=-\bar{C}^{T} \bar{C} .
\end{aligned}
$$

Equivalently, to compute the controllability and observability Gramians we can solve the continuous-time Lyapunov equations

$$
\begin{aligned}
& \underline{A} P \underline{E}^{T}+\underline{E} P \underline{A}^{T}=-\underline{B} \underline{B}^{T}, \\
& \underline{A}^{T} Q \underline{E}+\underline{E}^{T} Q \underline{A}=-\underline{C}^{T} \underline{C},
\end{aligned}
$$

where $\lambda \underline{A}-\underline{E}=: \lambda(\bar{A}-\bar{E})-(\bar{A}+\bar{E})$ is the Cayleytransformed pencil [25], and $\underline{B}=\sqrt{2} \bar{B}$, and $\underline{C}=\sqrt{2} \bar{C}$. Note that the solutions of (12) and (13) are identical to the solutions of (14) and (15), respectively [20, 25]. It is true that the Cayley transformation can destroy the system's sparsity pattern where it needs to be restored. But, using proper permutations and efficient computation, one can recover this difficulty.

Since both the Gramians are symmetric and positive semidefinite, they have symmetric Gramians factors

$$
\begin{gathered}
P=R R^{T}, \\
Q=L L^{T} .
\end{gathered}
$$

The main drawback of BT based model order reduction (MOR) techniques is the computation of the solutions of the Lyapunov equations that are used in computing the Gramian factors. During the recent decades several approaches have been developed that allow exploiting the fact that often all coefficient matrices are highly sparse and the numbers of inputs and outputs are very small compared to the number of DoFs. Then usually the Gramians $P$ and $Q$ can be expressed in low-rank factored forms; that is, $R$ and $L$ are thin rectangular matrices. Therefore instead of computing the full Gramian factors it is quite reasonable to compute the thin rectangular matrices $R$ and $L$.

2.4. Low-Rank ADI for LTI Discrete-Time Systems. One popular iterative method for computing the low-rank $R$ and $L$ is the generalized low-rank Cholesky factor-alternating directions implicit (GLR-ADI) iteration [11, 26, 27]. In the following we briefly introduce the GLR-ADI methods to solve continuoustime algebraic Lyapunov equations (14) and (15). For model reduction of system (1) we have to solve such a kind of Lyapunov equations. 
The GLR-ADI iterations successively compute the columns of the two Gramian factors following the simple steps [28]:

$$
\begin{aligned}
& Z_{1}=V_{1}=\sqrt{2\left|\operatorname{Re}\left(\mu_{1}\right)\right|}\left(F+\mu_{1} N\right)^{-1} G, \\
& V_{i}=\left[V_{i-1}-\left(\mu_{i}+\bar{\mu}_{i-1}\right)\left(F+\mu_{i} N\right)^{-1} N V_{i-1}\right], \\
& Z_{i}=\left[\begin{array}{ll}
Z_{i-1} & \sqrt{2\left|\operatorname{Re}\left(\mu_{i}\right)\right|} V_{i}
\end{array}\right], \quad i=2: i_{\max } .
\end{aligned}
$$

In this iteration either $(N, F, G)=(\underline{E}, \underline{A}, \underline{B})$ is applied to generates $Z=R^{T}$, or $(N, F, G)=\left(\underline{E}^{T}, \underline{A}^{T}, \underline{C}^{T}\right)$ is used for $Z=L^{T}$.

A set of optimal ADI shift parameters or simply shift parameters $\left\{\mu_{i}\right\}_{i=1}^{J} \subset \mathbb{C}^{-}$are necessary for fast convergence of the GLR-ADI iterations. We will discuss the shift parameter selection criterion later in this section. In these iterations we also see that if the maximum number of iterations $i_{\max }$ is greater than the number of shifts $J$, then the shift parameters are used in a cyclic way.

In the GLR-ADI iterations all of the input matrices $N$, $F$, and $G$ are real, due to the complex shift parameters in each iteration step, the updated $Z_{i}$ store complex data, which increases the overall complexity and memory requirements of the method. Moreover, in the balancing based methods using these complex Gramian factors yields complex reduced systems by performing some complex arithmetic operations. This problem is resolved in [22]. In this regard, the important assumption is that the selected ADI shift parameters should be proper.

Definition 3. The ADI shift parameters $\left\{\mu_{i}\right\}_{i=1}^{J} \subset \mathbb{C}^{-}$are called proper if $\mu_{i}$ and $\mu_{i+1}$ are complex conjugates of each other when one of them is complex.

In [22] it is shown that at the $(i+1)$-st iteration of the GLR-ADI iterations, $V_{i+1}$ can be computed by

$$
V_{i+1}=\bar{V}_{i}+2 \delta \operatorname{Im}\left(V_{i}\right)
$$

where $\delta=\operatorname{Re}\left(\mu_{i}\right) / \operatorname{Im}\left(\mu_{i}\right)$. This identity states that, for $\mu_{i+1}=$ $\bar{\mu}_{i}, V_{i+1}$ can be computed explicitly from $V_{i}$, which releases us from solving a shifted linear system with $F+\bar{\mu}_{i} N$. This idea also results in the following theorem.

Theorem 4. Let us assume a set of proper ADI shift parameters. For a pair of complex conjugate shifts $\left\{\mu_{i}, \mu_{i+1}:=\bar{\mu}_{i}\right\}$, the two subsequent block iterates $V_{i}$ and $V_{i+1}$ of GLR-ADI iterations satisfy [22]

$$
\begin{gathered}
{\left[V_{i}, V_{i+1}\right]=\left[\sqrt{-2 \operatorname{Re}\left(\mu_{i}\right)}\left(\operatorname{Re}\left(V_{i}\right)+\delta \operatorname{Im}\left(V_{i}\right)\right),\right.} \\
\left.\sqrt{-2 \operatorname{Re}\left(\mu_{i}\right)} \sqrt{\delta^{2}+1} \operatorname{Im}\left(V_{i}\right)\right] .
\end{gathered}
$$

This theorem reveals that for a pair of complex conjugate shifts at any iteration step in the GLR-ADI iteration $Z_{i}$ can be updated by

$$
\begin{gathered}
Z_{i+1}=\left[Z_{i-1}, \sqrt{-2 \operatorname{Re}\left(\mu_{i}\right)}\left(\operatorname{Re}\left(V_{i}\right)+\delta \operatorname{Im}\left(V_{i}\right)\right),\right. \\
\left.\sqrt{-2 \operatorname{Re}\left(\mu_{i}\right)} \sqrt{\delta^{2}+1} \operatorname{Im}\left(V_{i}\right)\right] .
\end{gathered}
$$

A version of the GLR-ADI algorithm is summarized in [29, Algorithm 5], which computes low-rank real Gramian factors. Additionally, the GLR-ADI iterations can be stopped whenever the norm of the ADI-residual

$$
\mathscr{F}\left(Z_{i}\right)=F_{i} Z_{i}^{T} N^{T}+N Z_{i} Z_{i}^{T} F^{T}+G G^{T}
$$

becomes very small. But computing $\left\|\mathscr{F}\left(Z_{i}\right)\right\|$ in Frobeniusnorm or 2-norm in each iteration step is an expensive task, since in each iteration the resulting residual (21) is an $n_{c} \times$ $n_{c}$ matrix. Recently, in [30] the authors show that, in the $i$ th iteration, the ADI-residual in (21) can be represented as

$$
\mathscr{F}\left(Z_{i}\right)=W_{i} W_{i}^{H},
$$

with

$$
W_{i}=\left(\prod_{j=1}^{i}\left(F-\bar{\mu}_{j} N\right)\left(F+\mu_{j} N\right)^{-1}\right) G,
$$

and the $V_{i}$ in the GLR-ADI iterations can be expressed as [30]

$$
V_{i}=\left(F+\mu_{i} N\right)^{-1} W_{i-1} .
$$

The authors in [30] also show that the ADI-residual factor defined in (23) can be computed by the expression

$$
W_{i}=W_{i-1}-2 \operatorname{Re}\left(\mu_{i}\right) N V_{i} .
$$

In the case of real setting when we consider $\mu_{i+1}:=\bar{\mu}_{i}$, one must compute

$$
W_{i+1}=W_{i-1}-4 \operatorname{Re}\left(\mu_{i}\right) N\left(\operatorname{Re}\left(V_{i}\right)+\delta \operatorname{Im}\left(V_{i}\right)\right),
$$

where $\delta$ is defined in (18). The rank of $W_{i}$ is at most $m_{c}$, that is, the number of columns of $G$. Therefore, the computation of the Frobenius-norm or 2-norm of $\left\|W_{i} W_{i}^{T}\right\|=\left\|W_{i}^{T} W_{i}\right\|$ in each iteration is extremely cheap. Applying these strategies (computation of real Gramian factors and low-rank residual based stopping techniques), the updated GLR-ADI will be briefly discussed in Section 3.

\section{Efficient Solutions of Causal LPDALEs Using Low-Rank ADI Methods}

The basic ADI method for linear systems has been proposed in [31] and then used in [11, 12, 17, 32-34] for solving (projected) continuous-time and discrete-time Lyapunov equations. Consider the LPDALE (9a), where the matrix $\mathbf{E}=\operatorname{diag}\left(E_{0}, \ldots, E_{K-1}\right)$ is singular. For such equations both the ADI and Smith iterations fail to converge [20]. This 
problem is circumvented by considering a generalized Cayley transformation [25]:

$$
\mathfrak{C}(\mathscr{E}, \mathscr{A})=\lambda(\mathbf{A}-\mathbf{E})-(\mathbf{A}+\mathbf{E})
$$

which transforms the LPDALE (9a) to an equivalent projected continuous-time algebraic Lyapunov equation (PCALE):

$$
\begin{aligned}
\mathscr{E} \mathbf{G}^{\mathrm{cr}} \mathscr{A}^{T}+\mathscr{A} \mathbf{G}^{\mathrm{cr}} \mathscr{E}^{T}=-2 \mathbf{P}_{l} \mathbf{B} \mathbf{B}^{T} \mathbf{P}_{l}^{T}, \\
\mathbf{G}^{\mathrm{cr}}=\mathbf{P}_{r} \mathbf{G}^{\mathrm{cr}} \mathbf{P}_{r}^{T} .
\end{aligned}
$$

Here $\lambda \mathscr{E}-\mathscr{A}=\lambda(\mathbf{A}-\mathbf{E})-(\mathbf{A}+\mathbf{E})$ is the Cayley-transformed pencil. In this transformation the finite stable eigenvalues of $\lambda \mathbf{E}-\mathbf{A}$ are mapped to the finite stable eigenvalues of $\lambda \mathscr{E}-\mathscr{A}$, and the infinite eigenvalue of $\lambda \mathbf{E}-\mathbf{A}$ is mapped to $\lambda=1$. More details of that transformation can be found in $[20,25]$. The solutions of PCALE (28) and (9a) are unique and they are solvable for every $\mathbf{B B}^{T}$ [20].

3.1. Efficient Computation of Low-Rank Gramian Factors. We present the updated version of low-rank ADI methods for Lyapunov equation (28), which is also the solution of (9a). An updated algorithm for the low-rank ADI method of generalized state space systems can be found in [29]. Here the idea is generalized for the periodic descriptor system.

Applying ADI method the solution $\mathbf{G}^{\mathrm{cr}}$ of Lyapunov equation (28) can be obtained by performing the following iteration steps, beginning with $\mathbf{G}_{0}^{\mathrm{cr}}=0$ and $i=1$ :

$$
\begin{aligned}
\left(\mathscr{A}+\mu_{i} \mathscr{E}\right)_{i} \mathbf{G}_{(i-1 / 2)}^{\mathrm{cr}}= & -\mathbf{G}_{i-1}^{\mathrm{cr}}\left(\mathscr{A}^{T}-\mu_{i} \mathscr{E}\right)-\widetilde{\mathscr{B}} \widetilde{\mathscr{B}}^{T} \\
\left(\mathscr{A}+\mu_{i} \mathscr{E}\right) \bar{\mu}_{i} \mathbf{G}_{(i-1 / 2)}^{\mathrm{cr}}= & -\mathbf{G}_{i-1 / 2}^{\mathrm{cr}}\left(\mathscr{A}^{T}-\mu_{i} \mathscr{E}\right) \\
& -\widetilde{\mathscr{B}} \widetilde{\mathscr{B}}^{T}
\end{aligned}
$$

where $\mu_{i} \in \mathbb{C}^{-}$, with $i=1,2, \ldots, i_{\text {max }}$, is a shift parameter and $\widetilde{\mathscr{B}}=\sqrt{2} \mathbf{P}_{l} \mathbf{B}$. Steps (29a) and (29b) can be written in a single step

$$
\mathbf{G}_{i}^{\mathrm{cr}}=\mathscr{A}_{\mu_{i}} \mathbf{G}_{i-1}^{\mathrm{cr}} \mathscr{A}_{\mu_{i}}^{*}-2 \operatorname{Re}\left(\mu_{i}\right) \widetilde{\mathscr{B}}_{\mu_{i}} \widetilde{\mathscr{B}}_{\mu_{i}}^{*}
$$

where $\mathscr{A}_{\mu_{i}}=\left(\mathscr{A}-\bar{\mu}_{i} \mathscr{E}\right)\left(\mathscr{A}+\mu_{i} \mathscr{E}\right)^{-1}$ and $\widetilde{\mathscr{B}}_{\mu_{i}}=\left(\mathscr{A}+\mu_{i} \mathscr{E}\right)^{-1} \widetilde{\mathscr{B}}$. The error bound in iteration $i$ is given by (see, e.g., [35])

$$
\Delta \mathbf{G}_{i}^{\mathrm{cr}}=\mathbf{G}^{\mathrm{cr}}-\mathbf{G}_{i}^{\mathrm{cr}}=\mathscr{A}_{\mu_{i}}\left(\mathbf{G}^{\mathrm{cr}}-\mathbf{G}_{i-1}^{\mathrm{cr}}\right) \mathscr{A}_{\mu_{i}}^{*}
$$

Since $\mathbf{G}_{0}^{\mathrm{cr}}=0$, this leads to

$$
\Delta \mathbf{G}_{i}^{\mathrm{cr}}=\mathbf{G}^{\mathrm{cr}}-\mathbf{G}_{i}^{\mathrm{cr}}=\prod_{l=1}^{i} \mathscr{A}_{\mu_{l}} \mathbf{G}^{\mathrm{cr}} \prod_{l=1}^{i} \mathscr{A}_{\mu_{l}}^{*}
$$

In $i$ th iteration the residual can be computed by

$$
\begin{aligned}
\operatorname{Res}_{i} & =\mathscr{A} \mathbf{G}_{i}^{\mathrm{cr}} \mathscr{E}^{T}+\mathscr{E} \mathbf{G}_{i}^{\mathrm{cr}} \mathscr{A}^{T}+\widetilde{\mathscr{B}} \widetilde{\mathscr{B}}^{T} \\
& =\mathscr{A}\left(\mathbf{G}_{i}^{\mathrm{cr}}-\mathbf{G}^{\mathrm{cr}}\right) \mathscr{E}^{T}+\mathscr{E}\left(\mathbf{G}_{i}^{\mathrm{cr}}-\mathbf{G}^{\mathrm{cr}}\right) \mathscr{A}^{T} \\
& =\prod_{l=1}^{i} \mathscr{A}_{\mu_{l}}\left(-\left(\mathscr{A}^{\mathrm{cr}} \mathscr{E}^{T}+\mathscr{E} \mathbf{G}^{\mathrm{cr}} \mathscr{A}^{T}\right)\right) \prod_{l=1}^{i} \mathscr{A}_{\mu_{l}}^{*} \\
& =\prod_{l=1}^{i} \mathscr{A}_{\mu_{l}} \widetilde{\mathscr{B}} \widetilde{\mathscr{B}}^{T} \prod_{l=1}^{i} \mathscr{A}_{\mu_{l}}^{*}=\mathscr{W}_{i} \mathscr{W}_{i}^{*},
\end{aligned}
$$

where $\mathscr{W}_{i}=\prod_{l=1}^{i} \mathscr{A}_{\mu_{l}} \widetilde{\mathscr{B}}$.

We observe that the computed $\mathbf{G}_{i}^{\mathrm{cr}}$ in (30) can be decomposed as

$$
\mathbf{G}_{i}^{\mathrm{cr}}=\mathscr{Z}_{i} \mathscr{E}_{i}^{*}
$$

where $\mathscr{Z}_{i} \in \mathbb{R}^{n \times k_{l}}$ and $k_{l} \ll n$. Since the number of inputs is very small compared to the dimension of the system, the right hand side of (9a) is a low-rank matrix. Therefore often this decomposition is possible, and $\mathscr{Z}_{i}$ can be computed by following iteration steps:

$$
\begin{aligned}
& \mathscr{Z}_{1}=\sqrt{2\left|\operatorname{Re}\left(\mu_{1}\right)\right|}\left(\mathscr{A}+\mu_{1} \mathscr{E}\right)^{-1} \widetilde{\mathscr{B}}, \\
& \mathscr{Z}_{i}=\left[\begin{array}{ll}
\mathscr{A}_{\mu_{i}} \mathscr{Z}_{i-1} & \sqrt{2\left|\operatorname{Re}\left(\mu_{i}\right)\right|}\left(\mathscr{A}+\mu_{i} \mathscr{E}\right)^{-1} \widetilde{\mathscr{B}}
\end{array}\right],
\end{aligned}
$$

with $i=2,3, \ldots, i_{\max }$, and $i_{\max }$ is maximum number of iterations. In each iteration in (35b) the number of columns of $\mathscr{Z}_{i}$ is increased by the number of columns $\mathscr{Z}_{i-1}$. Therefore after few steps the number of columns of $\mathscr{Z}_{i}$ grows dramatically. In order to resolve this drawback a further optimization occurs, where the number of columns to modify $\mathscr{Z}_{i}$ is kept constant by using the clever reordering of the ADI-parameters. This updated version of GLR-ADI [36] is given below:

$$
\begin{aligned}
& \mathscr{Z}_{1}=\mathscr{V}_{1}=\sqrt{2\left|\operatorname{Re}\left(\mu_{1}\right)\right|}\left(\mathscr{A}+\mu_{1} \mathscr{E}\right)^{-1} \widetilde{\mathscr{B}}, \\
& \mathscr{V}_{i}=\left[\mathscr{V}_{i-1}-\left(\mu_{i}+\bar{\mu}_{i-1}\right)\left(\mathscr{A}+\mu_{i} \mathscr{E}\right)^{-1} \mathscr{E} \mathscr{V}_{i-1}\right] \text {, }
\end{aligned}
$$

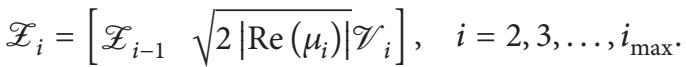

Unfortunately, the GLR-ADI iteration does not preserve the block diagonal structure at every iteration step in Algorithm 1 . But $\mathbf{G}_{i}^{\mathrm{cr}}=\mathscr{Z}_{i} \mathscr{Z}_{i}^{T}$ has almost block diagonal form since it approximates the solution of the LPDALE (9a) given by $\mathbf{G}^{\mathrm{cr}}=\operatorname{diag}\left(G_{1}^{\mathrm{cr}}, \ldots, G_{K-1}^{\mathrm{cr}}, G_{0}^{\mathrm{cr}}\right)$, where $G_{k}^{\mathrm{cr}}$ are the periodic solutions of (5). We then consider the block partitioning

$$
\mathscr{Z}_{i}=\left[R_{1 i}^{T}, \ldots, R_{K-1, i}^{T}, R_{0 i}^{T}\right]^{T},
$$

with $R_{k i} \in \mathbb{R}^{n_{k} \times r_{i}}$, and the causal reachability Gramian $G_{k}^{\mathrm{cr}}$ can be approximated by the matrices $R_{k i} R_{k i}^{T}$ for $k=0,1, \ldots, K-1$.

We compute low-rank Gramian factor $\mathscr{Z}$, so that $\mathscr{Z}^{T} \approx$ $\mathbf{G}^{\mathrm{cr}}$ by following the steps (36a)-(36c). We summarize the resulting iteration using column compression in Algorithm 1. 


\section{Input: $\mathscr{A}, \mathscr{E}, \widetilde{\mathscr{B}}$.}

Output: $\mathscr{Z}=\mathscr{E}_{i}$, such that $\mathbf{G}_{i}^{\mathrm{cr}} \approx \mathscr{Z}_{i} \mathscr{E}_{i}^{T}$.

(1) $\mathscr{W}_{0}=\widetilde{\mathscr{B}}, \mathscr{Z}_{0}=[], i=1$;

(2) $\mathscr{V}_{i}=\left(\mathscr{A}+\mu_{i} \mathscr{E}\right)^{-1} \mathscr{W}_{i-1}$;

(3) if $\operatorname{Im}\left(\mu_{i}\right)=0$ then

(4) $\mathscr{Z}_{i}=\left[\begin{array}{ll}\mathscr{Z}_{i-1} & \sqrt{-2 \mu_{i}} V_{i}\end{array}\right]$;

(5) $\quad \mathscr{W}_{i}=\mathscr{W}_{i-1}^{i-1}-2 \mu_{i} \mathscr{E} \mathscr{V}_{i}$.;

(6) else

(7) $\gamma=-2 \operatorname{Re}\left(\mu_{i}\right), \delta=\operatorname{Re}\left(\mu_{i}\right) / \operatorname{Im}\left(\mu_{i}\right)$;

(8) $\mathscr{Z}_{i+1}=\left[\begin{array}{lll}\mathscr{Z}_{i-1} & \sqrt{2 \gamma}\left(\operatorname{Re}\left(\mathscr{V}_{i}\right)+\delta \operatorname{Im}\left(\mathscr{V}_{i}\right)\right) & \sqrt{2 \gamma} \sqrt{\left(\delta^{2}+1\right)} \operatorname{Im}\left(\mathscr{V}_{i}\right)\end{array}\right]$;

(9) $\mathscr{W}_{i+1}=\mathscr{W}_{i-1}+2 \gamma \mathscr{E}\left(\operatorname{Re}\left(\mathscr{V}_{i}\right)+\delta \operatorname{Im}\left(\mathscr{V}_{i}\right)\right)$;

(10) $\quad i=i+1$;

(11) $i=i+1$;

Algorithm 1: Updated GLR-ADI (UGLR-ADI) for LPDALEs.

It should be noted that in Algorithm 1 the computation of the matrices $\mathscr{E}$ and $\mathscr{A}$ can be avoided by rewriting the GLR-ADI iteration in terms of the original matrices $\mathbf{E}$ and $\mathbf{A}[20]$. We should also note that we do not compute the inverses of the matrices at step (2) of Algorithm 1 explicitly. We represent the matrices $\mathscr{E}$ and $\mathscr{A}$ with the original sparse matrices with $\mathbf{E}$ and $\mathbf{A}$ and use the sparse inversion technique in step (2) [20].

The iteration can be stopped in the $i$ th step if the residual norm, that is, $\left\|\operatorname{Res}_{i}\right\|$, where $\operatorname{Res}_{i}$ is defined in (33), is sufficiently small. If we can compute $\mathscr{W}_{i}$ as a low-rank factor then computing $\|$ Res $\|$ is very cheap. Here, we discuss how to compute the low-rank residual-factor from each iteration in the GLRCF-ADI algorithm. Following the procedure as shown in Section 2.4, iterate $\mathscr{V}_{i}$ in (36b) can also be written as

$$
\begin{aligned}
\mathscr{V}_{i} & =\left(\mathscr{A}+\mu_{i} \mathscr{E}\right)^{-1}\left(\mathscr{A}-\bar{\mu}_{i-1} \mathscr{E}\right) \mathscr{V}_{i-1}=\left(\mathscr{A}+\mu_{i} \mathscr{E}\right)^{-1} \\
& \cdot\left(\mathscr{A}-\bar{\mu}_{i-1} \mathscr{E}\right)\left(\mathscr{A}+\mu_{i-1} \mathscr{E}\right)^{-1}\left(\mathscr{A}-\bar{\mu}_{i-2} \mathscr{E}\right) \mathscr{V}_{i-2} \\
= & \left(\mathscr{A}+\mu_{i} \mathscr{E}\right)^{-1} \prod_{l=1}^{i-1} \mathscr{A}_{\mu_{l}} \widetilde{\mathscr{B}}=\left(\mathscr{A}+\mu_{i} \mathscr{E}\right)^{-1} \mathscr{W}_{i-1},
\end{aligned}
$$

[using (33)]

Also,

$$
\begin{aligned}
\mathscr{W}_{i} & =\left(\mathscr{A}-\bar{\mu}_{i} \mathscr{E}\right)\left(\mathscr{A}+\bar{\mu}_{i} \mathscr{E}\right)^{-1}\left(\prod_{l=1}^{i-1} \mathscr{A}_{\mu_{l}}\right) \widetilde{\mathscr{B}} \\
& =\left(\mathscr{A}-\bar{\mu}_{i} \mathscr{E}\right)^{-1} \mathscr{V}_{i}=\left(\mathscr{A}-\bar{\mu}_{i} \mathscr{E}\right)\left(\mathscr{A}+\bar{\mu}_{i} \mathscr{E}\right) \mathscr{W}_{i-1} \\
& =\left(I-2 \operatorname{Re}\left(\mu_{i}\right) \mathscr{E}\left(\mathscr{E}+\mu_{i} \mathscr{A}\right)^{-1}\right) \mathscr{W}_{i-1}=\mathscr{W}_{i-1} \\
& -2 \operatorname{Re}\left(\mu_{i}\right) \mathscr{E} \mathscr{V}_{i}, \\
\mathscr{W}_{i+1} & =\mathscr{W}_{i}-2 \operatorname{Re}\left(\bar{\mu}_{i}\right) \mathscr{E} \mathscr{V}_{i+1}=\mathscr{W}_{i-1} \\
& -2 \operatorname{Re}\left(\mu_{i}\right) \mathscr{E} \mathscr{V}_{i} \\
& -2 \operatorname{Re}\left(\bar{\mu}_{i}\right) \mathscr{E}\left(\operatorname{Re}\left(\mathscr{V}_{i}\right)-j \operatorname{Im}\left(V_{i}\right)+2 \delta \operatorname{Im}\left(V_{i}\right)\right) \\
& =\mathscr{W}_{i-1}-2 \operatorname{Re}\left(\mu_{i}\right) \mathscr{E}\left(\operatorname{Re}\left(\mathscr{V}_{i}\right)-j \operatorname{Im}\left(\mathscr{V}_{i}\right)\right) \\
& -2 \operatorname{Re}\left(\bar{\mu}_{i}\right) \mathscr{E}\left(\operatorname{Re}\left(\mathscr{V}_{i}\right)-j \operatorname{Im}\left(\mathscr{V}_{i}\right)+2 \delta \operatorname{Im}\left(\mathscr{V}_{i}\right)\right) \\
& =\mathscr{W}_{i-1}-4 \operatorname{Re}\left(\mu_{i}\right) \mathscr{E}\left(\operatorname{Re}\left(\mathscr{V}_{i}\right)+\delta \operatorname{Im}\left(\mathscr{V}_{i}\right)\right) .
\end{aligned}
$$

3.2. Selection of the ADI Shift Parameters. The convergence rate of the low-rank $\mathrm{ADI}$ algorithms discussed above depends on a set of ADI shift parameters. The ADI shift parameters were originally introduced by Wachspress in [37] to solve Lyapunov equations using the ADI methods. The author shows that a set of optimal ADI shift parameters $\left\{\mu_{i}\right\}_{i=1}^{J}$ for system (2) can be computed by solving the so-called ADI minmax problem $[37,38]$ :

$$
\begin{aligned}
& \left\{\mu_{1}, \mu_{2}, \ldots, \mu_{i}\right\} \\
& =\underset{\mu_{1}, \mu_{2}, \ldots, \mu_{i} \in \mathbb{C}^{-} \backslash\{-1\}}{\arg \min } \max _{\lambda_{l} \in \operatorname{Sp}\left(\mathscr{C}^{-1} \mathscr{A}\right) \backslash\{1\}} \prod_{j=1}^{i} \frac{\left|\bar{\mu}_{j}-\lambda_{l}\right|}{\left|\mu_{j}+\lambda_{l}\right|},
\end{aligned}
$$

where $\operatorname{Sp}\left(\mathscr{E}^{-1} \mathscr{A}\right)$ is the spectrum of the matrix $\mathscr{E}^{-1} \mathscr{A}$.

For a large-scale system, determining the entire spectrum of $(\mathscr{A}, \mathscr{E})$ is almost impossible. Therefore, in the literature several techniques are proposed; see, for example, [26, 36, $39,40]$ to solve the min-max problem (40) using a much smaller part of the spectrum. Currently, one such commonly used technique is Penzl's heuristic approach introduced in [26], where $k_{+}$Ritz values (see, e.g., [41]) and $k_{-}\left(k_{+}, k_{-} \ll\right.$ $n)$ reciprocal Ritz values with respect to $\mathscr{E}^{-1} \mathscr{A}$ and $\mathscr{A}^{-1} \mathscr{E}$, respectively, are employed. A complete procedure of the heuristic approach can be found in [20]. However, for largescale descriptor systems, computing the Ritz values with respect to $\mathscr{E}^{-1} \mathscr{A}$ is a challenging task since $\mathscr{E}$ is then not invertible.

Another ADI shift selection criterion that we emphasize here is the adaptive approach which is introduced in [21]. The performances of this adaptive shift selection approach for a large-scale system are also investigated in [29, 42, 43]. There the authors show that the approach is superior to the heuristic approach, especially for the (generalized) descriptor systems. To initialize the shifts, we first compute the eigenvalues of the projected pencil

$$
\lambda \mathcal{U}^{T} \mathscr{E} \mathcal{U}-\mathscr{U}^{T} \mathscr{A} \mathcal{U}, \quad \lambda \in \mathbb{C},
$$

where $\mathcal{U}$ is formed by the basis of range of any $n_{c} \times r$ $\left(r \ll n_{c}\right)$ random matrix. Then select some desired number 
of optimal shifts by solving the ADI min-max problem like in the heuristic procedure. Then whenever all the initial shifts have been used, the pencil is projected to the span of the current $V_{i}$ (see, e.g., Algorithm 1 of [21]) and computes some optimal shifts from the eigenvalues of the projected pencil and uses them as the set of next shift parameters. This approach is repeated while the algorithm has not converged to the given tolerance.

\section{Smith Method for Noncausal LPDALEs}

Consider the LPDALE (9b). For nonsingular $\mathscr{A}$, this equation is equivalent to the LPDALE:

$$
\begin{array}{r}
\mathbf{G}^{\mathrm{ncr}}-\left(\mathbf{A}^{-1} \mathbf{E}\right) \mathbf{G}^{\mathrm{ncr}}\left(\mathbf{A}^{-1} \mathbf{E}\right)^{T}=\mathbf{Q}_{r} \mathbf{A}^{-1} \mathbf{B B}^{T} \mathbf{A}^{-T} \mathbf{Q}_{r}^{T}, \\
\mathbf{G}^{\mathrm{ncr}}=\mathbf{Q}_{r} \mathbf{G}^{\mathrm{ncr}} \mathbf{Q}_{r}^{T} .
\end{array}
$$

Note that if $\eta$ is the index of the system [20], then the approximate solution of the noncausal LPDALE (9b) can be obtained by the Smith iterations [14]:

$$
\begin{aligned}
\mathbf{G}_{\eta}^{\mathrm{ncr}} & =\sum_{j=0}^{\eta-1}\left(\mathbf{A}^{-1} \mathbf{E}\right)^{j} \mathbf{Q}_{r} \mathbf{A}^{-1} \mathbf{B B}^{T} \mathbf{A}^{-T} \mathbf{Q}_{r}^{T}\left(\left(\mathbf{A}^{-1} \mathbf{E}\right)^{T}\right)^{j} \\
& =\mathbf{G}^{\mathrm{ncr}} .
\end{aligned}
$$

Therefore, the Cholesky factor $X_{\eta}$ of the solution $\mathbf{G}^{\text {ncr }}=$ $X_{\eta} X_{\eta}^{T}$ of (42) and also of the LPDALE (9b) takes the form

$$
\begin{aligned}
X_{\eta} & =\left[\mathbf{Q}_{r} \mathbf{A}^{-1} \mathbf{B}, \mathbf{A}^{-1} \mathbf{E} \mathbf{Q}_{r} \mathbf{A}^{-1} \mathbf{B}, \ldots,\left(\mathbf{A}^{-1} \mathbf{E}\right)^{\eta-1}\right. \\
\cdot & \left.\mathbf{Q}_{r} \mathbf{A}^{-1} \mathbf{B}\right] .
\end{aligned}
$$

Thus, the solution of ( $9 \mathrm{~b})$ can be obtained with few computations for systems of low index. The solution of the noncausal LPDALE (9b) is briefly discussed in [20].

Similar to the causal case, we consider the block partitioning

$$
X_{\eta} X_{\eta}^{T}=\mathbf{G}^{\mathrm{ncr}}=\operatorname{diag}\left(G_{1}^{\mathrm{ncr}}, \ldots, G_{K-1}^{\mathrm{ncr}}, G_{0}^{\mathrm{ncr}}\right),
$$

such that the noncausal reachability Gramians of system (1) can be computed in factored form $G_{k}^{\text {ncr }}=\widetilde{X}_{k} \widetilde{X}_{k}^{T}$ for $k=$ $0,1, \ldots, K-1$.

Remark 5. The causal and noncausal observability Gramians $G_{k}^{\text {co }}$ and $G_{k}^{\text {nco }}$ of system (1) and the corresponding the lowrank Cholesky factors $L_{k}$ and $\tilde{Y}_{k}$, where $G_{k}^{\mathrm{co}} \approx L_{k} L_{k}^{T}$ and $G_{k}^{\text {nco }}=\tilde{Y}_{k} \tilde{Y}_{k}^{T}$, can also be determined from the corresponding LPDALEs that are dual to the LPDALEs (9a) and (9b); see [7] for details.

\section{Model Reduction of Periodic Systems}

For periodic descriptor system (1), we construct a reducedorder model of dimension $r$ of the form

$$
\begin{aligned}
\widetilde{E}_{k} \widetilde{x}_{k+1} & =\widetilde{A}_{k} \widetilde{x}_{k}+\widetilde{B}_{k} u_{k}, \\
\widetilde{y}_{k} & =\widetilde{C}_{k} \widetilde{x}_{k},
\end{aligned}
$$

\section{$k \in \mathbb{Z}$}

where $\widetilde{E}_{k} \in \mathbb{R}^{\gamma_{k+1} \times r_{k+1}}, \widetilde{A}_{k} \in \mathbb{R}^{\gamma_{k+1} \times r_{k}}, \widetilde{B}_{k} \in \mathbb{R}^{\gamma_{k+1} \times m_{k}}$, $\widetilde{C}_{k} \in \mathbb{R}^{q_{k} \times r_{k}}$ are $K$-periodic matrices, $\sum_{k=0}^{K-1} \gamma_{k}=\sum_{k=0}^{K-1} r_{k}=$ $r$, and $r \ll n$. Apart from having a much smaller state space dimension, the reduced-order model also preserves important physical properties of the original system such as regularity and stability and the small approximation error. Balanced truncation for discrete-time periodic descriptor system has been considered in [7, 20]. Some important concepts related to balanced truncation of discrete-time periodic descriptor systems has been presented in [8].

Consider $\left\{\left(E_{k}, A_{k}\right)\right\}_{k=0}^{K-1}$ is periodic stable, and the Cholesky factors of the causal and noncausal Gramians satisfy

$$
\begin{aligned}
G_{k}^{\mathrm{cr}} & =R_{k} R_{k}^{T}, \\
G_{k}^{\mathrm{co}} & =L_{k} L_{k}^{T}, \\
G_{k}^{\mathrm{ncr}} & =\widehat{R}_{k} \widehat{R}_{k}^{T}, \\
G_{k}^{\mathrm{nco}} & =\widehat{L}_{k} \widehat{L}_{k}^{T} .
\end{aligned}
$$

Then the causal and noncausal Hankel singular values $\sigma_{k, j}$ and $\theta_{k, j}$ of periodic descriptor system (1) are defined as

$$
\begin{aligned}
\sigma_{k, j} & =\zeta_{j}\left(L_{k}^{T} E_{k-1} R_{k}\right), \\
\theta_{k, j} & =\zeta_{j}\left(\widehat{L}_{k+1}^{T} A_{k} \widehat{R}_{k}\right),
\end{aligned}
$$

respectively, where $\zeta_{j}(\cdot)$ denotes the singular values of the corresponding product matrices. For a balanced system, one truncates the states related to the small causal Hankel singular values because they do not change system properties essentially. Unfortunately, truncation of small nonzero noncausal Hankel singular values may lead to unstable reduction [7].

Hence, we compute the singular value decompositions of the product matrices

$$
\begin{aligned}
& L_{k}^{T} E_{k-1} R_{k}=\left[U_{k, 1}, U_{k, 2}\right]\left[\begin{array}{cc}
\Sigma_{k, 1} & \\
& \Sigma_{k, 2}
\end{array}\right]\left[V_{k, 1}, V_{k, 2}\right]^{T}, \\
& \widetilde{Y}_{k+1}^{T} A_{k} \widetilde{X}_{k}=U_{k, 3} \Theta_{k} V_{k, 3}^{T},
\end{aligned}
$$

where $\left[U_{k, 1}, U_{k, 2}\right],\left[V_{k, 1}, V_{k, 2}\right], U_{k, 3}$, and $V_{k, 3}$ are orthogonal:

$$
\begin{aligned}
& \Sigma_{k, 1}=\operatorname{diag}\left(\sigma_{k, 1}, \ldots, \sigma_{k, r_{k}^{f}}\right), \\
& \Sigma_{k, 2}=\operatorname{diag}\left(\sigma_{k, r_{k}^{f}+1}, \ldots, \sigma_{k, n_{k}^{f}}\right),
\end{aligned}
$$




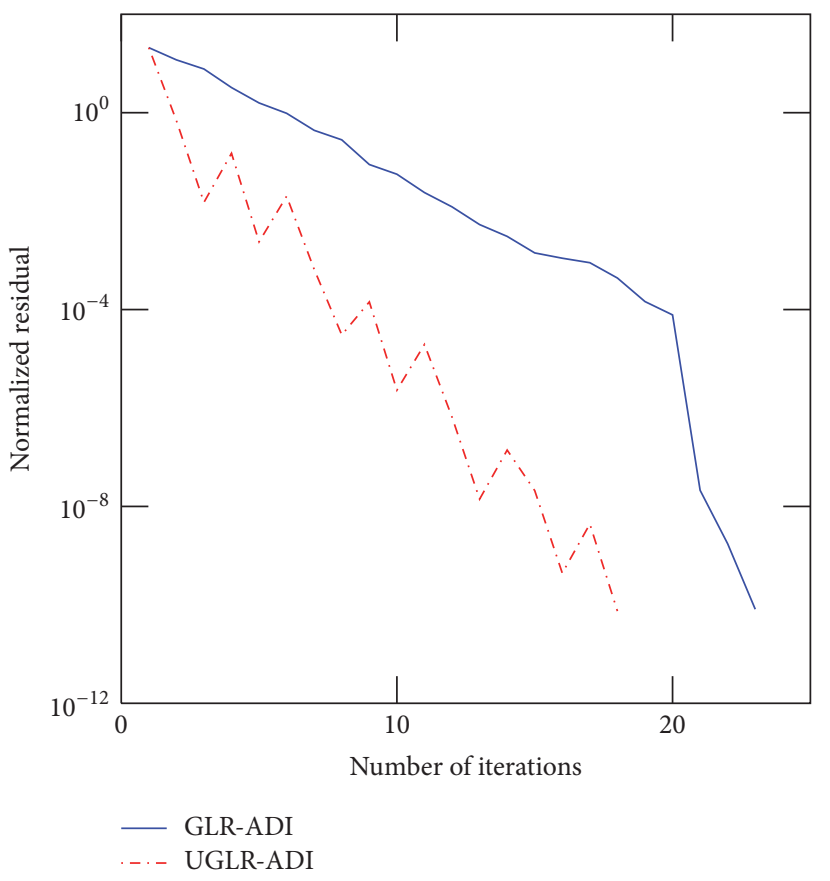

(a) Controllability Gramian factor

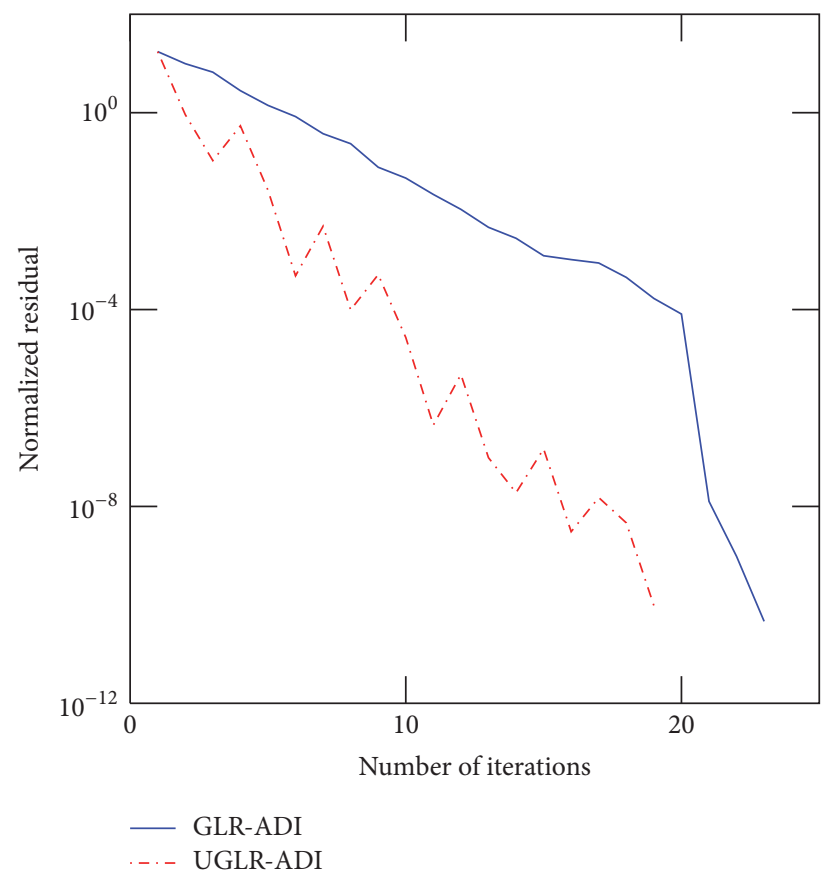

(b) Observability Gramian factor

FIGURE 1: Comparisons of the old algorithm (GLR-ADI) and new algorithm (UGLR-ADI) in terms of convergence rate in computing the low-rank Gramian factors.

with $\sigma_{k, 1} \geq \cdots \geq \sigma_{k, r_{k}^{f}}>\sigma_{k, r_{k}^{f}+1} \geq \cdots \geq \sigma_{k, n_{k}^{f}}$, and $\Theta_{k}=$ $\operatorname{diag}\left(\theta_{k, 1}, \ldots, \theta_{k, r_{k}^{\infty}}\right)$ is nonsingular for $k=0,1, \ldots, K-1$.

We then compute reduced-order system (46) as

$$
\begin{aligned}
\widetilde{E}_{k} & =S_{k, r}^{T} E_{k} T_{k+1, r}, \\
\widetilde{A}_{k} & =S_{k, r}^{T} A_{k} T_{k, r}, \\
\widetilde{B}_{k} & =S_{k, r}^{T} B_{k}, \\
\widetilde{C}_{k} & =C_{k} T_{k, r},
\end{aligned}
$$

where the projection matrices $S_{k, r}$ and $T_{k, r}$ have the form

$$
\begin{aligned}
& S_{k, r}=\left[L_{k+1} U_{k+1,1} \Sigma_{k+1,1}^{-1 / 2}, \widetilde{Y}_{k+1} U_{k, 3} \Theta_{k}^{-1 / 2}\right] \in \mathbb{R}^{\mu_{k+1} \times \gamma_{k+1}}, \\
& T_{k, r}=\left[R_{k} V_{k, 1} \Sigma_{k, 1}^{-1 / 2}, \widetilde{X}_{k} V_{k, 3} \Theta_{k}^{-1 / 2}\right] \in \mathbb{R}^{n_{k} \times r_{k}}
\end{aligned}
$$

with $r_{k}=r_{k}^{f}+r_{k}^{\infty}$ and $\gamma_{k+1}=r_{k+1}^{f}+r_{k}^{\infty}$.

Let $\mathscr{H}(z)=\mathscr{C}(z \mathscr{E}-\mathscr{A})^{-1} \mathscr{B}$ be the transfer function of lifted system (2) and $\widetilde{\mathscr{H}}(z)$ be the transfer function of the corresponding reduced-order lifted system formed from the reduced-order matrices in (51). Then the $\mathbb{H}_{\infty}$-norm error bound for the reduced-order system is given by

$$
\|\mathscr{H}-\widetilde{\mathscr{H}}\|_{\mathbb{H}_{\infty}} \leq 2 \operatorname{trace}\left(\operatorname{diag}\left(\Sigma_{0,2}, \ldots, \Sigma_{K-1,2}\right)\right),
$$

where $\Sigma_{k, 2}, k=0,1, \ldots, K-1$, contains the truncated causal Hankel singular values from (49).

\section{Numerical Experiments}

In this section, we present some numerical results. We consider an artificial periodic discrete-time descriptor system which is reformed from its original model in [8, Example 1]. The reformulation of the model is described in [20]. Here all the computations are carried out using MATLAB ${ }^{\circledR}$ R2012b on an Intel CORE i5 processor with a $2.90-\mathrm{GHz}$ clock and $16 \mathrm{~GB}$ of RAM.

To show the comparisons of GLR-ADI (i.e., Algorithm 2 in [20]) and our UGLR-ADI (i.e., Algorithm 1), we compute the low-rank Gramian factors by solving the LPDALEs applying both the algorithms. We also investigate the performances of both the heuristic and adaptive shifts to implement these algorithms. For implementing the GLR-ADI iteration we chose 40 optimal heuristic shifts out of 50 large and 40 small magnitude Ritz values. To select 40 heuristic shifts it takes 3.98 seconds of CPU time. In case of the adaptive shifts (for implementing UGLR-ADI method), in each cycle 40 proper shift parameters are selected following the procedure discussed above. We require only 0.03 seconds of CPU time to compute this number of adaptive shift parameters. Note that for computing the initial shifts first we project the pencil $(\mathscr{A}-\lambda \mathscr{E})$ onto the column space of an $n_{c} \times 100$ random matrix.

Figures 1 and 2, respectively, show the rate of convergence and the computational time of the GLR-ADI and UGLRADI methods. In both cases, we see that UGLR-ADI method performs better than the GLR-ADI method. We compute the reduced-order model of the periodic descriptor system by using the low-rank Gramian factors obtained from UGLRADI method. The reformed periodic descriptor system has 


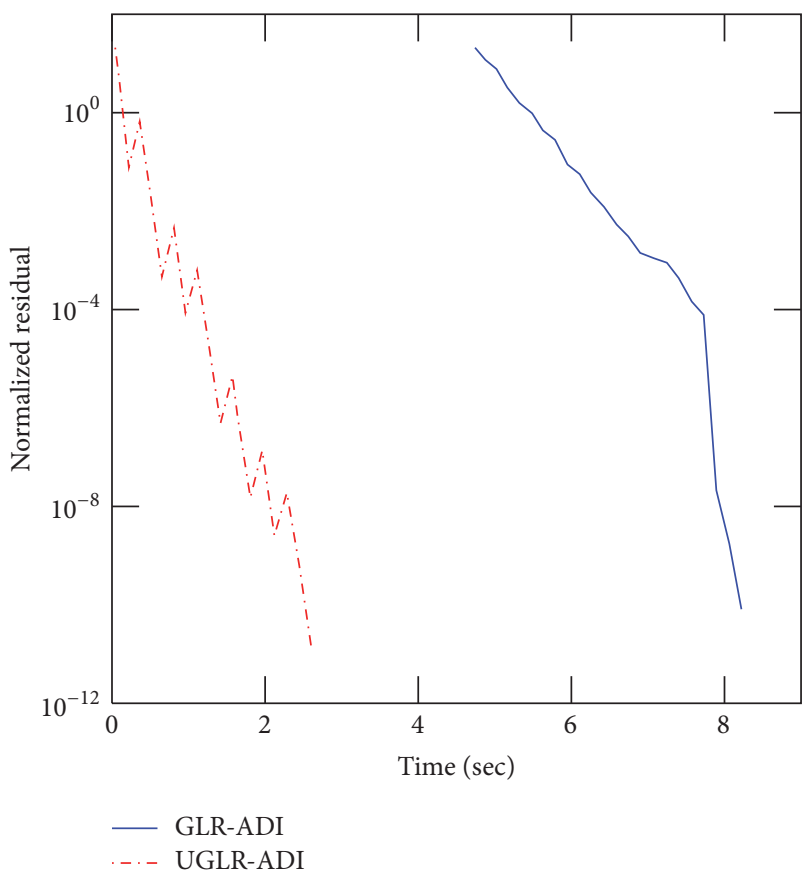

(a) Controllability Gramian factor

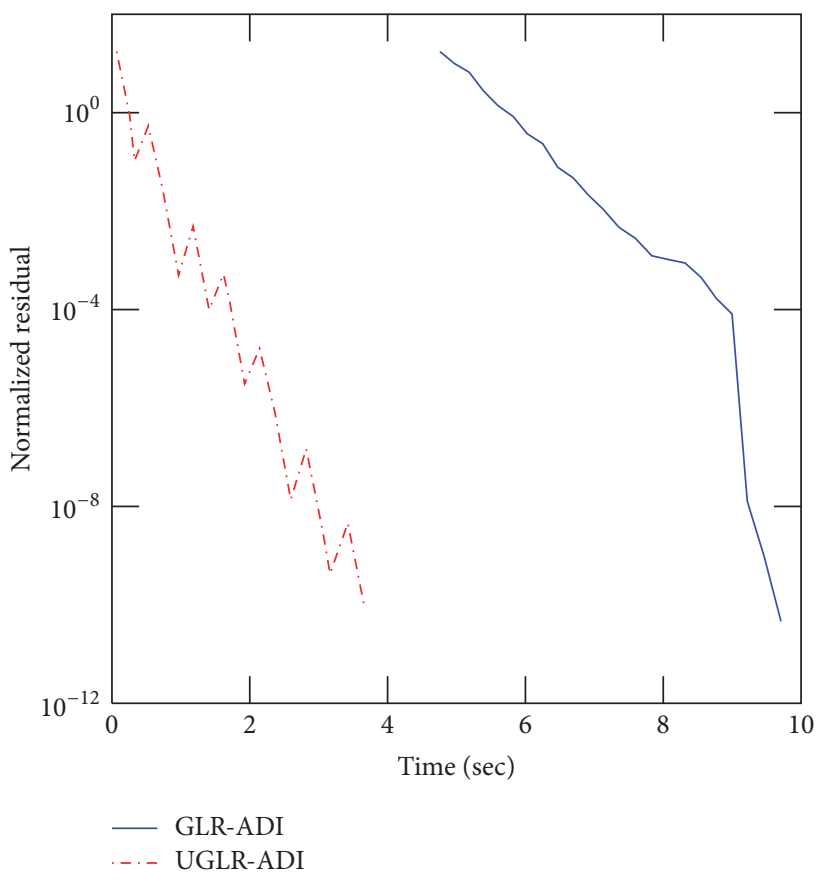

(b) Observability Gramian factor

FIGURE 2: Comparisons of the old algorithm (GLR-ADI) and new algorithm (UGLR-ADI) in terms of required time in computing the lowrank Gramian factors.

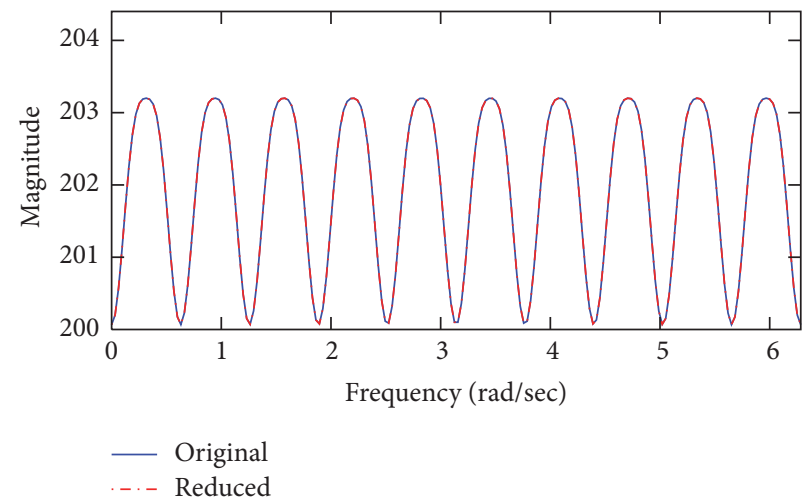

(a) Frequency responses

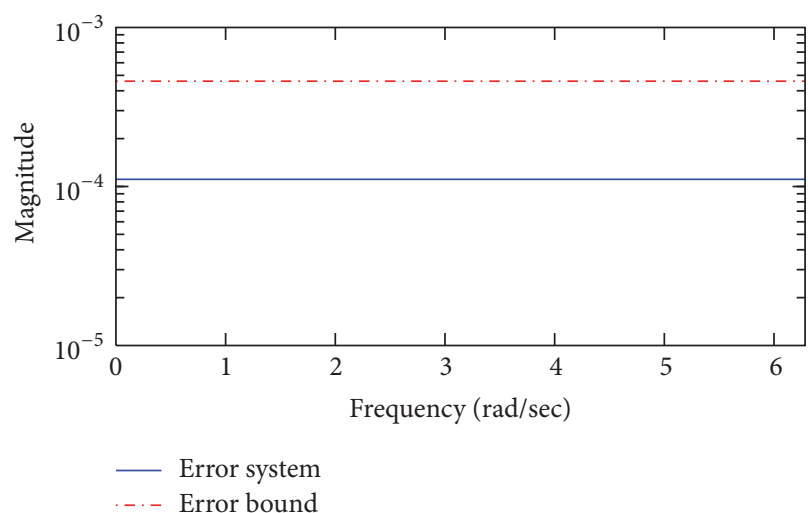

(b) Absolute error and error bound

FIGURE 3: Frequency responses and error bounds of the original and the reduced-order model.

$\mu_{k}=n_{k}=404, m_{k}=2$, and $q_{k}=3$ for $k=$ $0,1, \ldots, 9$. The set of periodic matrix pairs $\left\{\left(E_{k}, A_{k}\right)\right\}_{k=0}^{K-1}$ is periodic stable with $n_{k}^{f}=400$ and $n_{k}^{\infty}=4$ for $k=$ $0,1, \ldots, 9$. The original lifted system has order $n=4040$. The computed reduced-order model has subsystems of orders $(9,8,9,9,9,9,9,9,9,9)$. The comparisons of the frequency responses and the error bounds of the original model and the reduced model are shown in Figure 3. Figure 3(a) shows the frequency responses of the original and reduced models match nicely. The estimated error is below the error bound which is reflected in Figure 3(b).

\section{Conclusions}

In this article we have suggested the adaptive shifts based ADI method and reviewed the Smith method for computing the low-rank factors of the periodic Gramians for periodic discrete-time descriptor systems. Later, these factors are used in a balancing based model reduction technique to find a reduced-order model for the periodic discrete-time descriptor system. The proposed techniques save memory and the computational time to generate a reduced-order model. The capability and efficiency of the methods have been reflected in the numerical results. 


\section{Competing Interests}

The authors declare that there is no conflict of interests regarding the publication of this paper.

\section{References}

[1] S. Bittanti and P. Colaneri, "Invariant representations of discrete-time periodic systems," Automatica. A Journal of IFAC, the International Federation of Automatic Control, vol. 36, no. 12, pp. 1777-1793, 2000.

[2] M. Farhood, C. L. Beck, and G. E. Dullerud, "Model reduction of periodic systems: a lifting approach," Automatica, vol. 41, no. 6, pp. 1085-1090, 2005.

[3] P. Misra, "Time-invariant representation of discrete periodic systems," Automatica, vol. 32, no. 2, pp. 267-272, 1996.

[4] B. Park and E. Verriest, "Canonical forms of discrete linear periodically time-varying systems and a control application," in Proceedings of the 28th Conference on Decision and Control, pp. 1220-1225, Tampa, Fla, USA, December 1989.

[5] A. Varga, "Balancing related methods for minimal realization of periodic systems," Systems \& Control Letters, vol. 36, no. 5, pp. 339-349, 1999.

[6] A. Varga, "A periodic systems toolbox for MATLAB," in Proceedings of the 16th IFAC World Congress, Prague, Czech Republic, July 2005.

[7] P. Benner, M.-S. Hossain, and T. Stykel, "Model reduction of periodic descriptor systems using balanced truncation," in Model Reduction for Circuit Simulation, P. Benner, M. Hinze, and J. ter Maten, Eds., vol. 74 of Lecture Notes in Electrical Engineering, pp. 193-206, Springer, Berlin, Germany, 2011.

[8] E. K. Chu, H.-Y. Fan, and W.-W. Lin, "Projected generalized discrete-time periodic Lyapunov equations and balanced realization of periodic descriptor systems," SIAM Journal on Matrix Analysis and Applications, vol. 29, no. 3, pp. 982-1006, 2007.

[9] A. Varga, "An overview of recent developments in computational methods for periodic systems," in Proceedings of the 3rd IFAC Workshop "Periodic Control Systems" (PSYCO '07), pp. 157-162, St. Petersburg, Russia, August 2007.

[10] A. Varga and P. Van Dooren, "Computing the zeros of periodic descriptor systems," Systems \& Control Letters, vol. 50, no. 5, pp. 371-381, 2003.

[11] J.-R. Li and J. White, "Low rank solution of Lyapunov equations," SIAM Journal on Matrix Analysis and Applications, vol. 24, no. 1, pp. 260-280, 2002.

[12] T. Penzl, "A cyclic low rank Smith method for large sparse Lyapunov equations," SIAM Journal on Scientific Computing, vol. 21, pp. 1401-1418, 2000.

[13] S. Gugercin, D. C. Sorensen, and A. C. Antoulas, "A modified low-rank Smith method for large-scale Lyapunov equations," Numerical Algorithms, vol. 32, no. 1, pp. 27-55, 2003.

[14] R. Smith, "Matrix equation XA + BX = C," SIAM Journal on Applied Mathematics, vol. 16, pp. 198-201, 1968.

[15] K. Jbilou and A. J. Riquet, "Projection methods for large Lyapunov matrix equations," Linear Algebra and its Applications, vol. 415, no. 2-3, pp. 344-358, 2006.

[16] V. Simoncini, "A new iterative method for solving largescale Lyapunov matrix equations," SIAM Journal on Scientific Computing, vol. 29, no. 3, pp. 1268-1288, 2007.

[17] T. Stykel, "Low-rank iterative methods for projected generalized Lyapunov equations," Electronic Transactions on Numerical Analysis, vol. 30, pp. 187-202, 2008.
[18] T. Stykel and V. Simoncini, "Krylov subspace methods for projected Lyapunov equations," Applied Numerical Mathematics, vol. 62, no. 1, pp. 35-50, 2012.

[19] M.-S. Hossain, Numerical methods for model reduction of timevarying descriptor systems [Ph.D. thesis], Chemnitz University Of Technology, Chemnitz, Germany, 2011, http://nbn-resolving .de/urn:nbn:de:bsz:ch1-qucosa-74776.

[20] P. Benner, M.-S. Hossain, and T. Stykel, "Low-rank iterative methods for periodic projected Lyapunov equations and their application in model reduction of periodic descriptor systems," Numerical Algorithms, vol. 67, no. 3, pp. 669-690, 2014.

[21] P. Benner, J. Saak, and M. M. Uddin, "Balancing based model reduction for structured index-2 unstable descriptor systems with application to flow control," Numerical Algebra, Control and Optimization, vol. 6, no. 1, pp. 1-20, 2016.

[22] P. Benner, P. Kürschner, and J. Saak, "Efficient handling of complex shift parameters in the low-rank Cholesky factor ADI method," Numerical Algorithms, vol. 62, no. 2, pp. 225-251, 2013.

[23] E.-W. Chu, H.-Y. Fan, and W.-W. Lin, Reachability and Observability of Periodic Descriptor Systems, National Tsing Hua University, Hsinchu, Taiwan, 2005.

[24] M. Hinze and S. Volkwein, "Proper orthogonal decomposition surrogate models for nonlinear dynamical systems: error estimates and suboptimal control," in Dimension Reduction of Large-Scale Systems, P. Benner, V. Mehrmann, and D. Sorensen, Eds., vol. 45 of Lecture Notes in Computational Science and Engineering, pp. 261-306, Springer, Berlin, Germany, 2005.

[25] V. Mehrmann, "A step toward a unified treatment of continuous and discrete-time control problems," Linear Algebra and Its Applications, vol. 241-243, pp. 749-779, 1996.

[26] T. Penzl, "A cyclic low-rank Smith method for large sparse Lyapunov equations," SIAM Journal on Scientific Computing, vol. 21, no. 4, pp. 1401-1418, 2000.

[27] D. Sorensen and Y. Zhou, "Bounds on eigenvalue decay rates and sensitivity of solutions to Lyapunov equations," Tech. Rep. TR02-07, Department of Computational and Applied Mathematics, Rice University, Houston, Tex, USA, 2002, http:// www.caam.rice.edu/caam/trs/tr02.html\#TR02-07.

[28] P. Benner, J.-R. Li, and T. Penzl, "Numerical solution of large-scale Lyapunov equations, Riccati equations, and linearquadratic optimal control problems," Numerical Linear Algebra with Applications, vol. 15, no. 9, pp. 755-777, 2008.

[29] M. M. Uddin, Computational methods for model reduction of large-scale sparse structured descriptor systems [Ph.D. thesis], Otto von Guericke University Magdeburg, Magdeburg, Germany, 2015.

[30] P. Benner, P. Kürschner, and J. Saak, "An improved numerical method for balanced truncation for symmetric second-order systems," Mathematical and Computer Modelling of Dynamical Systems, vol. 19, no. 6, pp. 593-615, 2013.

[31] D. W. Peaceman and J. Rachford, "The numerical solution of parabolic and elliptic differential equations," Journal of the Society for Industrial and Applied Mathematics, vol. 3, pp. 2841, 1955.

[32] P. Benner and H. Faßbender, "On the numerical solution of large-scale sparse discrete-time Riccati equations," Advances in Computational Mathematics, vol. 35, no. 2-4, pp. 119-147, 2011.

[33] D. Calvetti, N. Levenberg, and L. Reichel, "Iterative methods for $X-A X B=C$," Journal of Computational and Applied Mathematics, vol. 86, no. 1, pp. 73-101, 1997. 
[34] A. Lu and E. L. Wachspress, "Solution of Lyapunov equations by alternating direction implicit iteration," Computers \& Mathematics with Applications, vol. 21, no. 9, pp. 43-58, 1991.

[35] F. D. Freitas, J. Rommes, and N. Martins, "Gramian-based reduction method applied to large sparse power system descriptor models," IEEE Transactions on Power Systems, vol. 23, no. 3, pp. 1258-1270, 2008.

[36] J. Saak, Efficient numerical solution of large scale algebraic matrix equations in PDE control and model order reduction [Ph.D. dissertation], TU Chemnitz, 2009.

[37] E. L. Wachspress, "Iterative solution of the Lyapunov matrix equation," Applied Mathematics Letters, vol. 1, no. 1, pp. 87-90, 1988.

[38] E. Wachspress, The ADI Model Problem, Springer, New York, NY, USA, 2013.

[39] J.-R. Li, Model reduction of large linear systems via low rank system gramians [Ph.D. thesis], Massachusettes Institute of Technology, 2000.

[40] P. Benner, H. Mena, and J. Saak, "On the parameter selection problem in the Newton-ADI iteration for large-scale Riccati equations," Electronic Transactions on Numerical Analysis, vol. 29, pp. 136-149, 2008.

[41] G. H. Golub and C. F. Van Loan, Matrix Computations, Johns Hopkins University Press, Baltimore, Md, USA, 3rd edition, 1996.

[42] P. Benner, J. Saak, and M. M. Uddin, "Structure preserving model order reduction of large sparse second-order index-1 systems and application to a mechatronics model," Mathematical and Computer Modelling of Dynamical Systems. Methods, Tools and Applications in Engineering and Related Sciences, vol. 22, no. 6, pp. 509-523, 2016.

[43] M. M. Uddin, M. S. Hossain, and M. F. Uddin, "Rational Krylov Subspace Method (RKSM) for solving the lyapunov equations of index-1 descriptor systems and application to balancing based model reduction," in Proceedings of the 9th International Conference on Electrical Computer Engineering (ICECE '16), pp. 451-454, Dhaka, Bangladesh, December 2016. 


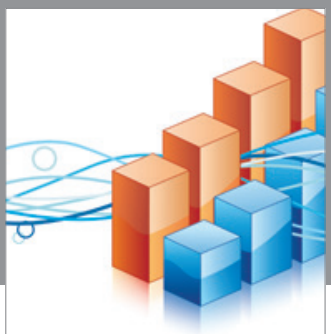

Advances in

Operations Research

vatem alat4

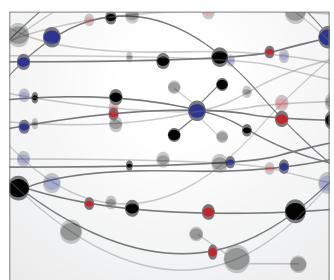

\section{The Scientific} World Journal
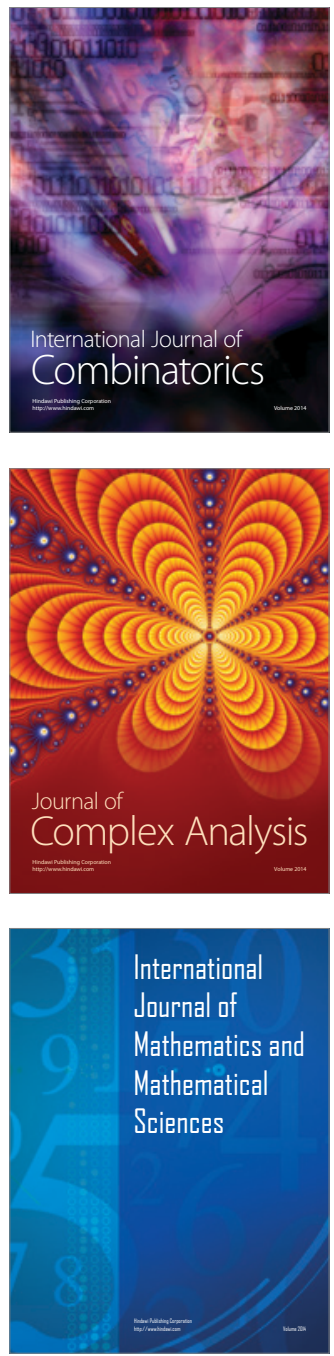
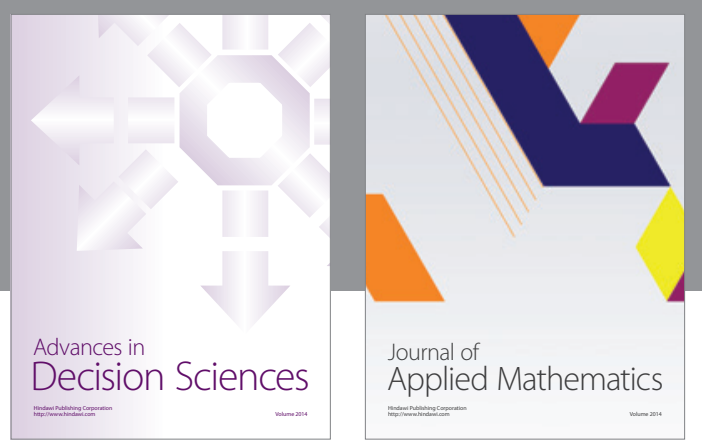

Algebra

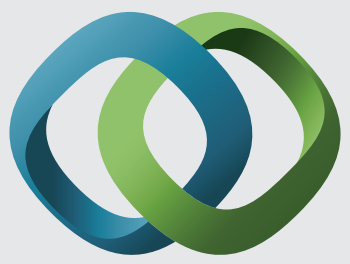

\section{Hindawi}

Submit your manuscripts at

https://www.hindawi.com
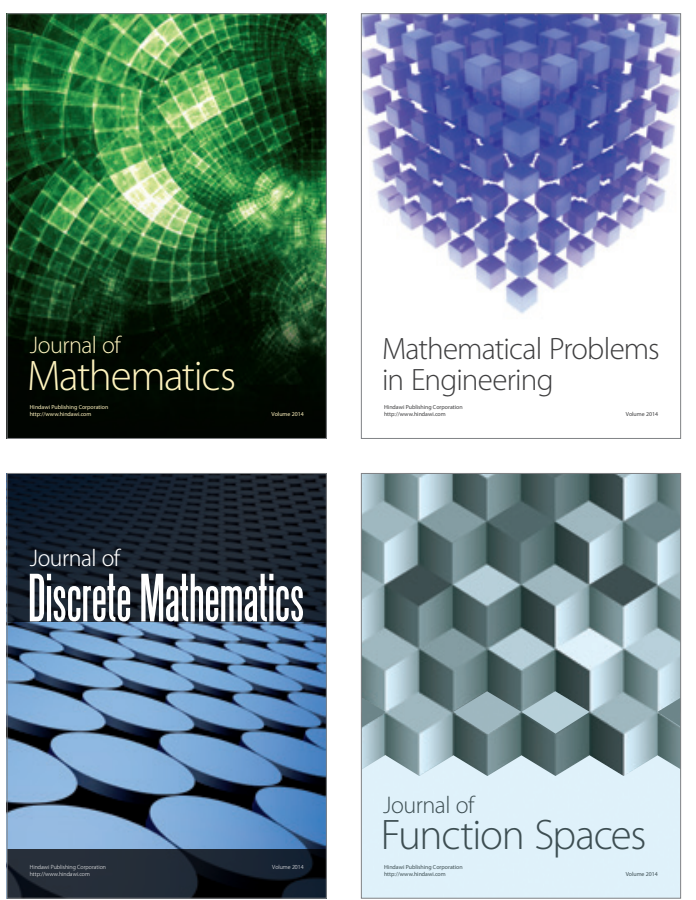

Mathematical Problems in Engineering
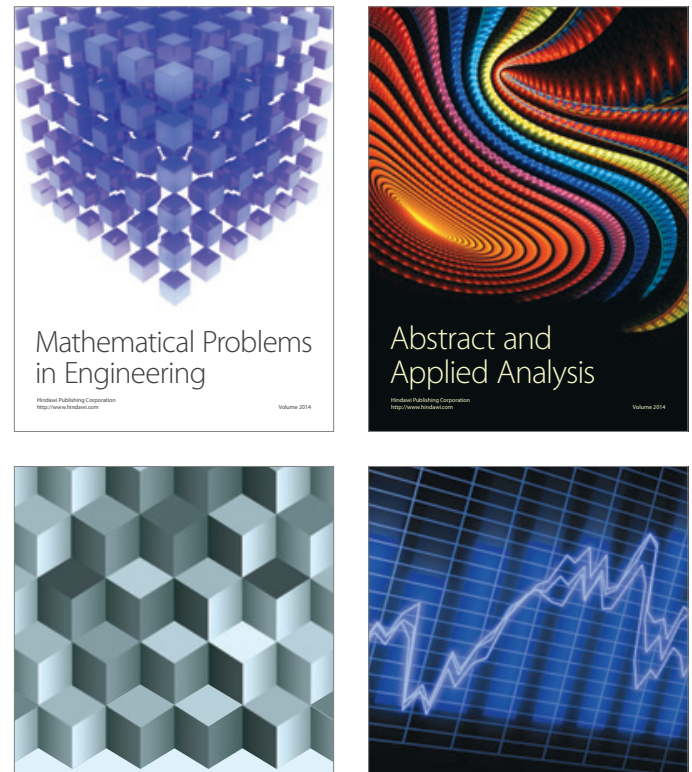

Journal of

Function Spaces

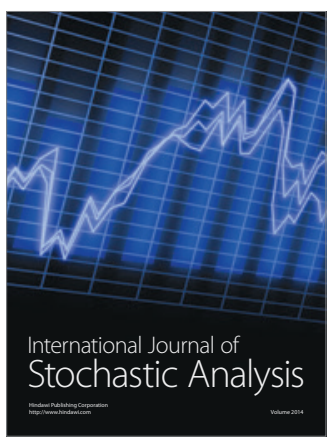

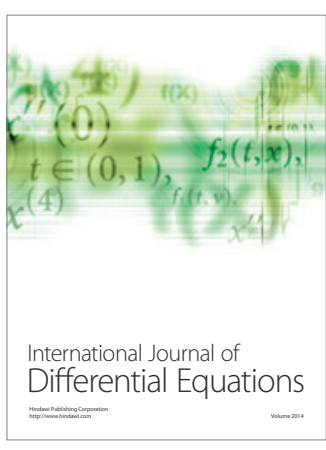
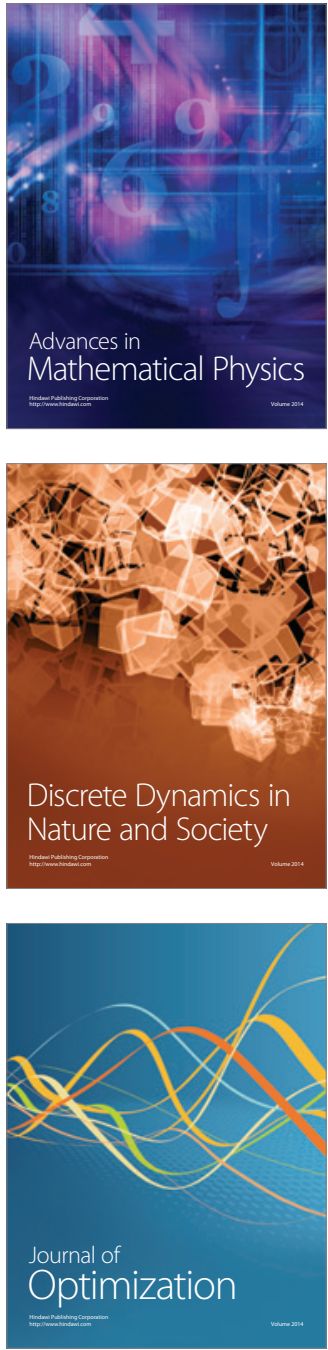